Kragujevac Journal of Mathematics

Volume 42(3) (2018), Pages 349-356.

\title{
SOME REFINEMENTS OF HERMITE-HADAMARD INEQUALITY AND AN OPEN PROBLEM
}

\author{
SLAVKO SIMIĆ ${ }^{1}$
}

\begin{abstract}
We presented here a refinement of Hermite-Hadamard inequality as a linear combination of its end-points. The problem of best possible constants is closely connected with well known Simpson's rule in numerical integration. It is solved here for a wide class of convex functions, but not in general. Some supplementary results are also given.
\end{abstract}

\section{INTRODUCTION}

A function $f: I \subset \mathbb{R} \rightarrow \mathbb{R}$ is said to be convex on an non-empty interval $I$ if the inequality

$$
f(p x+q y) \leq p f(x)+q f(y)
$$

holds for all $x, y \in I$ and all non-negative $p, q ; p+q=1$.

If the inequality (1.1) reverses, then $f$ is said to be concave on $I$ [1].

Let $f: I \subset \mathbb{R} \rightarrow \mathbb{R}$ be a convex function on an interval $I$ and $a, b \in I$ with $a<b$. Then

$$
f\left(\frac{a+b}{2}\right) \leq \frac{1}{b-a} \int_{a}^{b} f(t) d t \leq \frac{f(a)+f(b)}{2} .
$$

This double inequality is well known in the literature as Hermite-Hadamard integral inequality for convex functions. See, for example, [2] and references therein.

If $f$ is concave, both inequalities in (1.2) hold in the reversed direction.

Key words and phrases. Hermite-Hadamard integral inequality, differentiable function, convex function.

2010 Mathematics Subject Classification. Primary: 26D07. Secondary: 26D15.

Received: November 07, 2016.

Accepted: March 28, 2017. 
Our task in this paper is to improve the inequality (1.2) in a simple manner, i.e., to find some positive constants $\alpha, \beta, \gamma, \delta$ such that the relations

$$
\gamma(f(a)+f(b))+\delta f\left(\frac{a+b}{2}\right) \leq \frac{1}{b-a} \int_{a}^{b} f(t) d t \leq \alpha(f(a)+f(b))+\beta f\left(\frac{a+b}{2}\right)
$$

hold for any convex $f$.

Taking $f(t)=C t, C \in \mathbb{R} /\{0\}$, it can be easily seen that both conditions

$$
2 \alpha+\beta=1 ; \quad 2 \gamma+\delta=1,
$$

are necessary for (1.3) to hold.

Denote

$$
M(\gamma, \delta)=M_{f}(a, b ; \gamma, \delta):=\gamma(f(a)+f(b))+\delta f\left(\frac{a+b}{2}\right)
$$

and

$$
N(\alpha, \beta)=N_{f}(a, b ; \alpha, \beta):=\alpha(f(a)+f(b))+\beta f\left(\frac{a+b}{2}\right) .
$$

Since

$$
\begin{aligned}
N(\alpha, \beta) & =(2 \alpha)\left(\frac{f(a)+f(b)}{2}\right)+\beta f\left(\frac{a+b}{2}\right) \\
& \leq \max \left\{\frac{f(a)+f(b)}{2}, f\left(\frac{a+b}{2}\right)\right\}=\frac{f(a)+f(b)}{2},
\end{aligned}
$$

and, consequently

$$
\begin{aligned}
M(\gamma, \delta) & =(2 \gamma)\left(\frac{f(a)+f(b)}{2}\right)+\delta f\left(\frac{a+b}{2}\right) \\
& \geq \min \left\{\frac{f(a)+f(b)}{2}, f\left(\frac{a+b}{2}\right)\right\}=f\left(\frac{a+b}{2}\right),
\end{aligned}
$$

it follows that the inequality (1.3) represents a refinement of Hermite-Hadamard inequality (1.2).

Now, it can be seen that the bound $M(0,1)$ is best possible in general case. Indeed, let $\gamma \in(0,1 / 2]$ be fixed and the relation

$$
M_{f}(0,1 ; \gamma, \delta) \leq \int_{0}^{1} f(t) d t
$$

holds for arbitrary convex $f$.

Then the convex function $f(t)=t^{1 / \gamma}$ gives a counter-example.

This means that the left-hand side of Hermite-Hadamard inequality cannot be improved, in general, by the form of (1.3).

Nevertheless, such improvement is possible for some special classes of convex functions (see Corollary 2.1 below). 
The case of the bound $N(\alpha, \beta)$ is significantly harder. We found value $N(1 / 4,1 / 2)$ for which the right-hand side of (1.3) holds for any integrable convex function. Since $N(\alpha, \beta)$ is monotone increasing in $\alpha$, because

$$
\frac{d}{d \alpha} N_{f}(a, b ; \alpha, \beta)=f(a)+f(b)-2 f\left(\frac{a+b}{2}\right) \geq 0,
$$

it follows that the right-hand side of (1.3) also holds for $\alpha \in[1 / 4,1 / 2]$.

In search for best possible constants, note that

$$
\alpha \geq \frac{\frac{1}{b-a} \int_{a}^{b} f(t) d t-f\left(\frac{a+b}{2}\right)}{f(a)+f(b)-2 f\left(\frac{a+b}{2}\right)}:=F_{f}(a, b)
$$

and, if $f \in C^{\infty}(I)$ and $f^{\prime \prime}(t)>0$ for $t \in I$, then $\lim _{b \rightarrow a} F_{f}(a, b)=1 / 6$, independently of $f$.

Therefore, we obtain a potentially best possible bound $N(1 / 6,2 / 3)$. Unfortunately, as is shown in Theorem 2.3, this bound is best possible only for the class of differentiable convex functions for which $f^{(4)}(t) \geq 0, t \in I$.

Hence, we can formulate the following.

Open Question: find the best possible bound $N\left(\alpha^{*}, \beta^{*}\right)$ valid for all convex mappings from the class $C^{\infty}(I)$.

Since the function $g(t)=\frac{4 t^{3} \sqrt{t}}{35}-\frac{t^{4}}{12}$, convex on $I=[0,1]$, gives the value $\alpha=$ $.18128>1 / 6$, we have that $\alpha^{*} \in[.18128, .25]$.

\section{Results And Proofs}

We shall begin with the basic contribution to the problem defined above.

Theorem 2.1. Let $f: I \subset \mathbb{R} \rightarrow \mathbb{R}$ be a convex function on an interval $I$ and $a, b \in I$ with $a<b$. Then

$$
\frac{1}{b-a} \int_{a}^{b} f(t) d t \leq \frac{1}{4}(f(a)+f(b))+\frac{1}{2} f\left(\frac{a+b}{2}\right):=N(1 / 4,1 / 2) .
$$

Proof. We shall derive the proof by Hermite-Hadamard inequality itself. Indeed, applying twice the right part of this inequality, we get

$$
\frac{2}{b-a} \int_{a}^{\frac{a+b}{2}} f(t) d t \leq \frac{1}{2}\left(f(a)+f\left(\frac{a+b}{2}\right)\right)
$$

and

$$
\frac{2}{b-a} \int_{\frac{a+b}{2}}^{b} f(t) d t \leq \frac{1}{2}\left(f\left(\frac{a+b}{2}\right)+f(b)\right) .
$$

Summing, the result appears. Therefore, HH inequality has a self-improving property. 
For the sake of further refinements, we shall consider in the sequel functions from the class $C^{(m)}(I)$ i.e., functions which are continuously differentiable up to $\mathrm{m}$-th order on an interval $I \subset \mathbb{R}$.

We give firstly a sharp improvement of the result from Theorem 2.1.

Theorem 2.2. Let $f \in C^{(2)}(I)$ be convex on I together with its second derivative. Then for each $a, b \in I, a<b$,

$$
\frac{(b-a)^{2}}{48} f^{\prime \prime}\left(\frac{a+b}{2}\right) \leq N(1 / 4,1 / 2)-\frac{1}{b-a} \int_{a}^{b} f(t) d t \leq \frac{(b-a)^{2}}{96}\left[f^{\prime \prime}(a)+f^{\prime \prime}(b)\right] .
$$

If $f$ is convex and $f^{\prime \prime}$ concave on $I$, then

$$
\frac{(b-a)^{2}}{96}\left[f^{\prime \prime}(a)+f^{\prime \prime}(b)\right] \leq N(1 / 4,1 / 2)-\frac{1}{b-a} \int_{a}^{b} f(t) d t \leq \frac{(b-a)^{2}}{48} f^{\prime \prime}\left(\frac{a+b}{2}\right) .
$$

Proof. We need the following two assertions.

Lemma 2.1. [3] If $h$ is convex on $I=[a, b]$ and, for $x, y \in I, x+y=a+b$, then

$$
2 h\left(\frac{a+b}{2}\right) \leq h(x)+h(y) \leq h(a)+h(b) .
$$

Remark 2.1. Note that this result is a pre-HH inequality, i.e., $\mathrm{HH}$ inequality is its direct consequence. Indeed, let $x=p a+q b, y=q a+p b$ for $p, q \geq 0, p+q=1$. Then $x, y \in I$ and $x+y=a+b$. Hence,

$$
2 h\left(\frac{a+b}{2}\right) \leq h(p a+q b)+h(q a+p b) \leq h(a)+h(b) .
$$

Integrating this expression over $p \in[0,1]$ we obtain the very $\mathrm{HH}$ inequality.

Lemma 2.2. Let $f \in C^{(2)}(I)$ and $a, b \in I, a<b$. Then the following identity holds.

$$
N(1 / 4,1 / 2)-\frac{1}{b-a} \int_{a}^{b} f(t) d t=\frac{(b-a)^{2}}{16} \int_{0}^{1} t(1-t)\left[f^{\prime \prime}(x)+f^{\prime \prime}(y)\right] d t,
$$

with $x:=a \frac{t}{2}+b\left(1-\frac{t}{2}\right), y:=b \frac{t}{2}+a\left(1-\frac{t}{2}\right)$.

It is not difficult to prove this identity by double partial integration of its right-hand side.

Since $x+y=a+b$ and $f^{\prime \prime}$ is convex/concave, applying Lemma 2.1 the proof readily follows.

Another improvement of $\mathrm{HH}$ inequality is given in the next

Theorem 2.3. Let $f \in C^{(4)}(I)$ and $a, b \in I, a<b$. If $f, f^{\prime \prime}$ are convex on $I$, then

$$
\frac{1}{b-a} \int_{a}^{b} f(t) d t \leq N(1 / 6,2 / 3),
$$


and the coefficients $1 / 6,2 / 3$ are best possible for this class of functions.

If $f^{\prime \prime}$ is concave on $I$ then the reversed inequality takes place.

Proof. Note that the coefficients $1 / 6$ and 2/3 are involved in well-known Simpson's rule which is of importance in numerical integration. It says that

Lemma 2.3. [4] For an integrable $f$, we have

$$
\int_{x_{1}}^{x_{3}} f(t) d t=\frac{1}{3} h\left(f_{1}+4 f_{2}+f_{3}\right)-\frac{1}{90} h^{5} f^{(4)}(\xi),\left(x_{1}<\xi<x_{3}\right),
$$

where $f_{i}=f\left(x_{i}\right)$ and $h:=x_{2}-x_{1}=x_{3}-x_{2}$.

Now, taking $x_{1}=a, x_{2}=(a+b) / 2, x_{3}=b$, we get $h=(b-a) / 2$. Also, convexity/concavity of $f^{\prime \prime}$ on $I$ implies that $f^{(4)}(\xi) \gtrless 0$ and the proof follows.

Combining the second part of this theorem with the result of Theorem 2.1, we get

Corollary 2.1. For $f \in C^{(4)}(I)$ let $f$ be convex and $f^{\prime \prime}$ concave on $I$. Then

$$
N(1 / 6,2 / 3) \leq \frac{1}{b-a} \int_{a}^{b} f(t) d t \leq N(1 / 4,1 / 2)
$$

which gives a proper answer, regarding this class of functions, to the problem posed in Introduction.

Further refinement of the assertion from Theorem 2.3 is possible.

Theorem 2.4. For $f \in C^{(4)}(I)$ let $f$ and $f^{\prime \prime}$ be convex on $I$. Then

$$
\begin{aligned}
0 & \leq \frac{1}{6}[f(a)+f(b)]+\frac{2}{3} f\left(\frac{a+b}{2}\right)-\frac{1}{b-a} \int_{a}^{b} f(t) d t \\
& \leq \frac{(b-a)^{2}}{324}\left[f^{\prime \prime}(a)+f^{\prime \prime}(b)-2 f^{\prime \prime}\left(\frac{a+b}{2}\right)\right] .
\end{aligned}
$$

If $f$ is convex and $f^{\prime \prime}$ concave on $I$, then

$$
\begin{aligned}
0 & \leq \frac{1}{b-a} \int_{a}^{b} f(t) d t-\frac{1}{6}\left[f(a)+f(b)+4 f\left(\frac{a+b}{2}\right)\right] \\
& \leq \frac{(b-a)^{2}}{324}\left[2 f^{\prime \prime}\left(\frac{a+b}{2}\right)-\left(f^{\prime \prime}(a)+f^{\prime \prime}(b)\right)\right]
\end{aligned}
$$

The above theorem tightly refines Simpson's rule for this class of functions.

Proof. The left part is proved in Theorem 2.3. For the right part we shall use an integral identity. 
Lemma 2.4. For $f \in C^{(2)}(I)$, we have

$$
N(1 / 6,2 / 3)-\frac{1}{b-a} \int_{a}^{b} f(t) d t=\frac{(b-a)^{2}}{48} \int_{0}^{1} t(2-3 t)\left[f^{\prime \prime}(x)+f^{\prime \prime}(y)\right] d t,
$$

where $x$ and $y$ are the same as in Lemma 2.2 .

Writing,

$$
\int_{0}^{1} t(2-3 t)[\cdot] d t=\int_{0}^{2 / 3} t(2-3 t)[\cdot] d t-\int_{2 / 3}^{1} t(3 t-2)[\cdot] d t
$$

and applying Lemma 2.1 to each integral separately, the result appears since

$$
\int_{0}^{2 / 3} t(2-3 t) d t=\int_{2 / 3}^{1} t(3 t-2) d t=\frac{4}{27}
$$

Remark 2.2. Note that the convexity condition on $f$ in last three theorems is superfluous. It is stated there just to keep the connection with Hermite-Hadamard inequality.

\section{Applications in Means theory}

A mean is a map $M: \mathbb{R}_{+} \times \mathbb{R}_{+} \rightarrow \mathbb{R}_{+}$, with a property

$$
\min \{a, b\} \leq M(a, b) \leq \max \{a, b\}
$$

for each $a, b \in \mathbb{R}_{+}$.

Hence $M$ is necessary reflexive, $M(a, a)=a$.

Most known ordered family of means is the following family $\Delta$ of elementary means,

$$
\Delta: H \leq G \leq L \leq I \leq A \leq S
$$

where

$$
\begin{gathered}
H=H(a, b):=2(1 / a+1 / b)^{-1} ; \quad G=G(a, b):=\sqrt{a b} ; \quad L=L(a, b):=\frac{b-a}{\log b-\log a} ; \\
I=I(a, b):=\frac{1}{e}\left(b^{b} / a^{a}\right)^{1 /(b-a)} ; \quad A=A(a, b):=\frac{a+b}{2} ; \quad S=S(a, b):=a^{\frac{a}{a+b}} b^{\frac{b}{a+b}},
\end{gathered}
$$

are the harmonic, geometric, logarithmic, identric, arithmetic and Gini mean, respectively.

As an illustration of our results, we shall give in the sequel some sharp approximations of logarithmic and identric means. 
Theorem 3.1. The inequality $G \leq L \leq A$ can be improved to

$$
\frac{1}{3}(A+2 G)-\frac{2}{81}\left(\frac{A-G}{L}\right)^{2}(A+G) \leq L \leq \frac{1}{3}(A+2 G) .
$$

Similarly, an approximation of $1 / L$ in terms of the arithmetic and harmonic means is given by

$$
\frac{A-H}{6 A^{2}} \leq \frac{1}{2}\left(\frac{1}{A}+\frac{1}{H}\right)-\frac{1}{L} \leq \frac{A(A-H)}{6 H^{2}}\left(\frac{4}{H}-\frac{3}{A}\right)
$$

Proof. Applying Theorem 2.4 with $f=e^{t}$, we obtain

$$
\begin{aligned}
0 & \leq \frac{1}{6}\left(e^{x}+e^{y}\right)+\frac{2}{3} e^{\frac{x+y}{2}}-\frac{e^{x}-e^{y}}{x-y} \\
& \leq \frac{(x-y)^{2}}{324}\left(e^{x}+e^{y}-2 e^{\frac{x+y}{2}}\right) .
\end{aligned}
$$

Since $x$ and $y$ are arbitrary real numbers, putting $x=\log b, y=\log a$, we get

$$
\begin{aligned}
0 & \leq \frac{1}{3}(A+2 G)-L \leq \frac{(\log b-\log a)^{2}}{162}(A-G) \\
& =\frac{4}{162}\left(\frac{\log b-\log a}{b-a}\right)^{2}\left(A^{2}-G^{2}\right)(A-G)=\frac{2}{81}\left(\frac{A-G}{L}\right)^{2}(A+G),
\end{aligned}
$$

and the proof is done.

For the second part, applying Theorem 2.2 with $f=1 / t, f^{\prime \prime}=2 / t^{3}$, we get

$$
\frac{(b-a)^{2}}{24} \frac{1}{A^{3}} \leq \frac{1}{4}\left(\frac{1}{a}+\frac{1}{b}\right)+\frac{1}{2 A}-\frac{1}{L} \leq \frac{(b-a)^{2}}{48}\left(\frac{1}{a^{3}}+\frac{1}{b^{3}}\right) .
$$

Now, the identities $1 / a+1 / b=2 / H,(b-a)^{2}=4 A(A-H), A H=G^{2}$ yields the proof.

Finally, we shall give some interesting inequalities for the identric mean.

Theorem 3.2. For arbitrary positive $a, b$ we have

$$
\begin{gathered}
A^{2 / 3} G^{1 / 3} \leq I \leq A^{2 / 3} G^{1 / 3} \exp \left(\frac{(A-H)^{2}}{162 H}\left(\frac{1}{A}+\frac{2}{H}\right)\right) \\
A^{4 / 3}(a, b) S^{2 / 3}(a, b) \exp \left(-\frac{4}{81} \frac{(A(a, b)-H(a, b))^{2}}{A(a, b) H(a, b)}\right) \leq I\left(a^{2}, b^{2}\right) \leq A^{4 / 3}(a, b) S^{2 / 3}(a, b) .
\end{gathered}
$$

Proof. Applying Theorem 2.4 with $f=-\log t$, we obtain the proof.

For the second part, for $f=t \log t$, we get

$$
\frac{1}{b-a} \int_{a}^{b} f(t) d t=\frac{1}{4}\left(\frac{b^{2} \log b^{2}-a^{2} \log a^{2}}{b-a}-(a+b)\right)=\frac{a+b}{4} \log I\left(a^{2}, b^{2}\right) .
$$

Since $f^{\prime \prime}=1 / t$, Theorem 2.4 yields 


$$
\begin{aligned}
& \frac{1}{6}(a \log a+b \log b)+\frac{2}{3} A \log A-\frac{(b-a)^{2}}{324}\left(\frac{1}{a}+\frac{1}{b}-\frac{2}{A}\right) \\
& \leq \frac{a+b}{4} \log I\left(a^{2}, b^{2}\right) \leq \frac{1}{6}(a \log a+b \log b)+\frac{2}{3} A \log A,
\end{aligned}
$$

and the proof follows by dividing the last expression with $a+b=2 A$.

Acknowledgements. The author is grateful to the referee for his/her valuable comments.

\section{REFERENCES}

[1] G. H. Hardy, J. E. Littlewood and G. Polya, Inequalities, Cambridge University Press, Cambridge, 1978.

[2] C. P. Niculescu and L. E. Persson, Old and new on the Hermite-Hadamard inequality, Real Anal. Exchange 29(2) (2003), 663-685.

[3] S. Simic, On a convexity property, Krag. J. Math. 40(2) (2016), 166-171.

[4] C. W. Ueberhuber, Numerical Computation 2, Berlin, Springer-Verlag, 1997.

${ }^{1}$ Mathematical Institute SANU

KneZa Mihaila 36

11000 BeLgrade

SERBIA

E-mail address: ssimic@turing.mi.sanu.ac.rs 